\title{
The Hierarchy-of-Hypotheses Approach: A Synthesis Method for Enhancing Theory Development in Ecology and Evolution
}

\author{
TINA HEGER, CARLOS A. AGUILAR-TRIGUEROS, ISABELLE BARTRAM, RAUL RENNÓ BRAGA, GREGORY P. DIETL, \\ MARTIN ENDERS, DAVID J. GIBSON, LORENA GÓMEZ-APARICIO, PIERRE GRAS, KURT JAX, SOPHIE LOKATIS, \\ CHRISTOPHER J. LORTIE, ANNE-CHRISTINE MUPEPELE, STEFAN SCHINDLER, JOSTEIN STARRFELT, \\ ALEXIS D. SYNODINOS, AND JONATHAN M. JESCHKE
}

\begin{abstract}
In the current era of Big Data, existing synthesis tools such as formal meta-analyses are critical means to handle the deluge of information. However, there is a need for complementary tools that help to (a) organize evidence, (b) organize theory, and (c) closely connect evidence to theory. We present the hierarchy-of-hypotheses $(\mathrm{HoH})$ approach to address these issues. In an HoH, hypotheses are conceptually and visually structured in a hierarchically nested way where the lower branches can be directly connected to empirical results. Used for organizing evidence, this tool allows researchers to conceptually connect empirical results derived through diverse approaches and to reveal under which circumstances hypotheses are applicable. Used for organizing theory, it allows researchers to uncover mechanistic components of hypotheses and previously neglected conceptual connections. In the present article, we offer guidance on how to build an HoH, provide examples from population and evolutionary biology and propose terminological clarifications.
\end{abstract}

Keywords: hierarchy-of-hypotheses approach, knowledge synthesis, theory development, structuring ideas, linking evidence to theory

\section{n many disciplines, the volume of evidence published}

in scientific journals is steadily increasing. In principle, this increase should make it possible to describe and explain complex systems in much greater detail than ever before. However, an increase in available information does not necessarily correspond to an increase in knowledge and understanding (Jeschke et al. 2019). Publishing results in scientific journals and depositing data in public archives does not guarantee their practical application, reuse, or the advancement of theory. We suggest that this situation can be improved by the development, establishment, and regular application of methods that have the explicit aim of linking evidence and theory.

An important step toward more efficiently exploiting results from case studies is synthesis (for this and other key terms, see box 1). There is a wealth of methods available for statistically combining the results of multiple studies (Pullin et al. 2016, Dicks et al. 2017). These methods enable the synthesis of research results stemming from different studies that address a common question (Koricheva et al. 2013). In the environmental sciences, evidence synthesis has increased both in frequency and importance (Lortie 2014), seeking to make empirical evidence readily available and more suitable as a basis for decision-making (e.g., evidence-based decision making; Sutherland 2006, Diefenderfer et al. 2016, Pullin et al. 2016, Cook et al. 2017, Dicks et al. 2017). Moreover, methodological guidelines have been developed, and web portals implemented to collect and synthesize the results of primary studies. Prime examples are the platforms www.conservationevidence. com and www.environmentalevidence.org, alongside the European Union-funded projects EKLIPSE (www.eklipsemechanism.eu) and BiodiversityKnowledge (Nesshöver et al. 2016). These initiatives have promoted significant advances in the organization and assessment of evidence and the implementation of synthesis, thus allowing for a comprehensive representation of applied knowledge in environmental sciences.

BioScience 71: 337-349. (C) The Author(s) 2020. Published by Oxford University Press on behalf of the American Institute of Biological Sciences. This is an Open Access article distributed under the terms of the Creative Commons Attribution Non-Commercial License (http://creativecommons.org/licenses/ by-nc/4.0/), which permits non-commercial re-use, distribution, and reproduction in any medium, provided the original work is properly cited. For commercial re-use, please contact journals.permissions@oup.com doi:10.1093/biosci/biaa130 


\section{Box 1. Glossary.}

Evidence. Available body of data and information indicating whether a belief or proposition is true or valid (Howick 2011, Mupepele et al. 2016). These data and information can, for example, stem from an empirical observation, model output, or simulation.

Hypothesis. An assumption that (a) is based on a formalized or nonformalized theoretical model of the real world and (b) can deliver one or more testable predictions (after Giere et al. 2005).

Mechanistic hypothesis. Narrowed version of an overarching hypothesis, resulting from specialization or decomposition of the unspecified hypothesis with respect to assumed underlying causes.

Operational hypothesis. Narrowed version of an overarching hypothesis, accounting for a specific study design. Operational hypotheses explicate which method (e.g., which study system or research approach) is used to study the overarching hypothesis.

Overarching hypothesis. Unspecified assumption derived from a general idea, concept or major principle (i.e., from a general theoretical model).

Prediction. Statement about how data (i.e., measured outcome of an experiment or observation) should look if the underlying hypothesis is true.

Synthesis. Process of identifying, compiling and combining relevant knowledge from multiple sources.

Theory. A high-level—that is, general-system of conceptual constructs or devices to explain and understand ecological, evolutionary or other phenomena and systems (adapted from Pickett et al. 2007). Theory can consist of a worked out, integrated body of mechanistic rules or even natural laws, but it may also consist of a loose collection of conceptual frameworks, ideas and hypotheses.

Fostering evidence-based decision-making is crucial to solving specific applied problems. However, findings resulting from these applied approaches for evidence synthesis are usually not reconnected to a broader body of theory. Therefore, they do not consistently contribute to a structured or targeted advancement of theory-for example, by assessing the usefulness of ideas. It is a missed opportunity to not feed this synthesized evidence back into theory. A similar lack of connection to theory has been observed for studies addressing basic research questions (e.g., Jeltsch et al. 2013, Scheiner 2013). Evidence feeding back into theory, subsequently leading to further theory development, would become a more appealing, simpler and, therefore, more common process if there were well described and widely accepted methods. A positive example in this respect is structural equation modeling, especially if combined with metamodels (Grace et al. 2010). With this technique, theoretical knowledge directly feeds into mathematical models, and empirical data are then used to select the model best matching the observations.

In the present article, we provide a detailed description of a relatively new synthesis method-the hierarchy-ofhypotheses (HoH) approach (Jeschke et al. 2012, Heger et al. 2013)-that is complementary to existing knowledge synthesis tools. This approach offers the opportunity to organize evidence and ideas, and to create and display links between single study results and theory. We suggest that the representation of broad ideas as nested hierarchies of hypotheses can be powerful and can be used to more efficiently connect single studies to a body of theory. Empirical studies usually formulate very specific hypotheses, derive predictions from these about expected data, and test these predictions in experiments or observations. With an $\mathrm{HoH}$, it can be made explicit which broader ideas these specific hypotheses are linked to. The specific hypotheses can be characterized and visualized as subhypotheses of a broader idea or theory. Therefore, it becomes clear that the single study, although necessarily limited in its scope, is testing an important aspect of a broader idea or theory. Similarly, an $\mathrm{HoH}$ can be used to organize a body of literature that is too heterogeneous for statistical meta-analysis. It can be linked with a systematic review of existing studies, so that the studies and their findings are organized and hierarchically structured, thus visualizing which aspects of an overarching question or hypothesis each study is addressing. Alternatively, the $\mathrm{HoH}$ approach can be used to refine a broad idea on theoretical grounds and to identify different possibilities of how an idea, concept, or hypothesis can become more specific, less ambiguous, and better structured. Taken together, the approach can help to strengthen the theoretical foundations of a research field.

In this context, it is important to clarify what is meant by hypothesis. In the present article, we apply the terminology offered by the philosopher of science Ronald Giere and colleagues (Giere et al. 2005, see also Griesemer 2018). Accordingly, a hypothesis provides the connection of the (formalized or nonformalized) theoretical model that a researcher has, describing how a specific part of the world works in theory, to the real world by asserting that the model fits that part of the world in some specified aspect. A hypothesis needs to be testable, thus allowing the investigation of whether the theoretical model actually fits the real world. This is done by deriving one or more predictions from the hypothesis that state how data (gathered in an observation or experiment) should look if the hypothesis is true. 
The HoH approach has already been introduced as a tool for synthesis in invasion ecology (Jeschke et al. 2012, Heger et al. 2013, Heger and Jeschke 2014, Jeschke and Heger 2018a). So far, however, explicit and consistent guidance on how to build a hierarchy of hypotheses has not been formally articulated. The primary objective of this publication therefore is to offer a concrete, consistent, and refined description for those who want to use this tool or want to adopt it to their discipline. Furthermore, we want to stimulate methodological discussions about its further development and improvement. In the following, we outline the main ideas behind the $\mathrm{HoH}$ approach and the history of its development, present a primer for creating $\mathrm{HoHs}$, provide examples for applications within and outside of invasion ecology, and discuss its strengths and limitations.

\section{The hierarchy-of-hypotheses approach}

The basic tenet behind the $\mathrm{HoH}$ approach is that complexity can often be handled by hierarchically structuring the topic under study (Heger and Jeschke 2018c). The approach has been developed to clarify the link between big ideas, and experiments or surveys designed to test them. Usually, experiments and surveys actually test predictions derived from smaller, more specific ideas that represent an aspect or one manifestation of the big idea. Different studies all addressing a joint major hypothesis consequently often each address different versions of it. This diversity makes it hard to reconcile their results. The $\mathrm{HoH}$ approach addresses this challenge by dividing the major hypothesis into more specific formulations or subhypotheses. These can be further divided until the level of refinement allows for direct empirical testing. The result is a tree that visually depicts different ways in which a major hypothesis can be formulated. The empirical studies can then be explicitly linked to the branch of the tree they intend to address, thus making a conceptual and visual connection to the major hypothesis. Hierarchical nestedness therefore allows one to structure and display relationships between different versions of an idea, and to conceptually collate empirical tests addressing the same overall question with divergent approaches. A hierarchical arrangement of hypotheses has also been suggested by Pickett and colleagues (2007) in the context of the method of pairwise alternative hypothesis testing (or strong inference, Platt 1964). However, we are not aware of studies that picked up on or further developed this idea.

The $\mathrm{HoH}$ approach in its first version (Jeschke et al. 2012, Heger et al. 2013, Heger and Jeschke 2014) was not a formalized method with a clear set of rules on how to proceed. It emerged and evolved during a literature synthesis project through dealing with the problem of how to merge results of a set of highly diverse studies without losing significant information on what precisely these studies were addressing. In that first iteration of the $\mathrm{HoH}$ method, the branches of the hierarchy were selected by the respective author team, on the basis of expert knowledge and assessment of published data. Therefore, pragmatic questions guided the creation of the
$\mathrm{HoH}$ (e.g., which kind of branching helps group studies in a way that enhances interpretation?). Through further work on the approach, helpful discussions with colleagues, and critical comments (Farji-Brener and Amador-Vargas 2018, Griesemer 2018, Scheiner and Fox 2018), suggestions for its refinement were formulated (Heger and Jeschke 2018b, 2018c). The present article amounts to a further step in the methodological development and refinement of the $\mathrm{HoH}$ approach, including terminological clarifications and practical suggestions.

\section{A primer for building a hierarchy of hypotheses}

With the methodological guidance provided in the following, we take the initial steps toward formalizing the application of the $\mathrm{HoH}$ approach. However, we advocate that its usage should not be confined by rules that are too strict. Although we appreciate the advantages of strict methodological guidelines, such as those provided by The Collaboration for Environmental Evidence (2018) for synthesizing evidence in systematic reviews, we believe that when it comes to conceptual work and theory development, room is needed for creativity and methodological flexibility.

Applying the $\mathrm{HoH}$ approach involves four steps (figure 1). We distinguish two basic aims for creating an $\mathrm{HoH}$ : organizing evidence and organizing theory. These basic aims reflect the distinction between empirical and theoretical modeling approaches in Griesemer (2013). Creating and displaying links between evidence and theory can be part of the process in either case. In the first case (i.e., if the aim is to organize evidence), the process starts with a diverse set of empirical results and the question of how these can be grouped to enhance their joint interpretation or further analysis. In the second case (i.e., if the aim is to organize theory), the process of creating the hierarchy starts with decomposing an overarching hypothesis. An $\mathrm{HoH}$ allows one to make the meaning of this overarching hypothesis more explicit by formulating its components as separate subhypotheses from which testable, specific predictions can be derived.

Step 1. The starting point for an $\mathrm{HoH}$-based analysis in both cases, for organizing evidence as well as for organizing theory, is the identification of a focal hypothesis. This starting point is followed by the compilation of information (step 1 in figure 1). Which information needs to be compiled depends on whether the aim is structuring and synthesizing empirical evidence provided by a set of studies (e.g., Jeschke and Heger 2018a and example 1 below) or whether the research interest is more in the theoretical structure and subdivision of the overarching hypothesis (see examples 2 and 3 below). The necessary information needs to be gathered by means of a literature review guided by expert knowledge. Especially if the aim is to organize evidence, we recommend applying a standardized procedure (e.g., PRISMA, Moher et al. 2015, or ROSES, Haddaway et al. 2018) and recording the performed steps. 


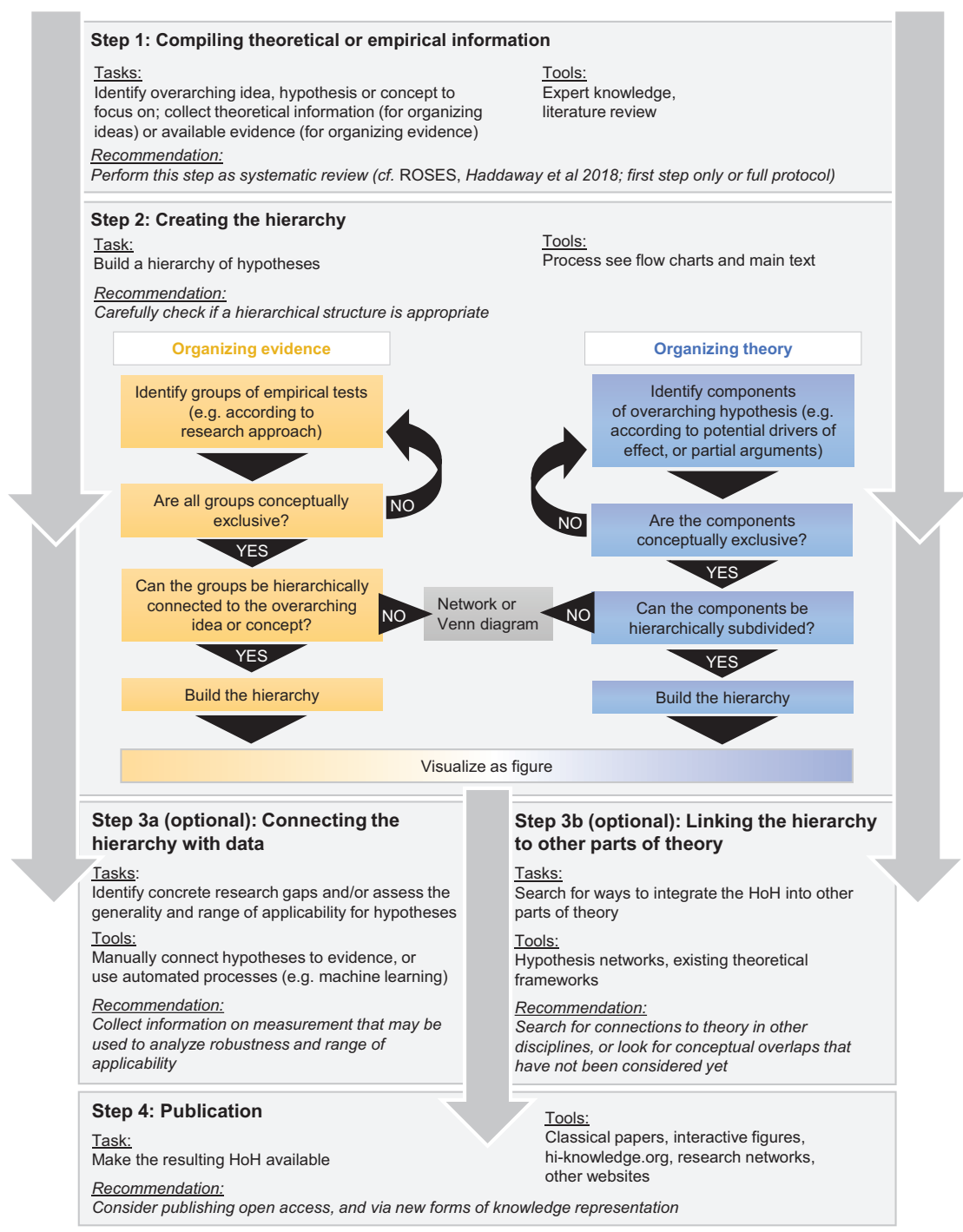

Figure 1. Workflow for the creation of a hierarchy of hypotheses. For a detailed explanation, see the main text.

Step 2. The next step is to create the hierarchy (step 2 in figure 1). If the aim is to organize evidence, step 1 will have led to the compilation of a set of studies empirically addressing the overarching hypothesis or a sufficiently homogeneous overarching theoretical framework. In step 2, these studies will need to be grouped. Depending on the aim of the study, it can be helpful to group the empirical tests of the overarching hypothesis according to study system (e.g., habitat, taxonomic group) or research approach (e.g., measured response variable). For example, in tests of the biotic resistance hypothesis in invasion ecology, which posits that an ecosystem with high biodiversity is more resistant against nonnative species than an ecosystem with lower biodiversity, Jeschke and colleagues (2018a) grouped empirical tests according to how the tests measured biodiversity and resistance against nonnative species. Some tests measured biodiversity as species richness, others as evenness or functional richness. The groups resulting from such considerations can be interpreted as representing operational hypotheses, because they specify the general hypothesis by accounting for diverse research approaches-that is, options for measuring the hypothesized effect (see also Griesemer 2018, Heger and Jeschke 2018c, as well as figure $2 \mathrm{a}$ and example 1 below). In such cases, we recommend displaying all resulting subhypotheses, if feasible.

If the aim is to organize theory, the overarching hypothesis is split into independent components on the basis of conceptual considerations (figure $2 \mathrm{~b}$ and 2c). This splitting of the overarching hypothesis can be done by creating branches according to which factors could have caused the respective process or pattern (see example 2 below, figure $2 b$ ).

Broad, overarching hypotheses often consist of several complementary partial arguments that are necessary elements. Consider the question why species often are well adapted to their biotic environment. A common hypothesis suggests that enduring interaction with enemies drives evolutionary changes, thus leading to adaptations of prey to their enemies (see example 3). This hypothesis presupposes that species face increasing risks from enemies but also that species' traits evolve in response to the changed risk (figure $2 \mathrm{c}$ and example 3 below). Decomposing overarching hypotheses into their partial arguments by formulating separate mechanistic hypotheses can enhance conceptual clarity and elucidate that sometimes, studies combined under one header are in fact addressing very different things.

For any type of branching, it is critical to identify components or groups (i.e., branches) that are mutually exclusive and not overlapping, so that an unambiguous assignment of single cases or observations into a box (i.e., subhypothesis) can be possible. If this is not feasible, it may be necessary to use conceptual maps, networks or Venn diagrams rather than hierarchical structures (figure 1, step 2; also see supplemental table S1). Therefore, care should be taken not to impose a hierarchical structure in cases where it is not helpful.

For many applications, the process of building an $\mathrm{HoH}$ can stop at this step, and a publication of the results can be considered (step 4). The resulting $\mathrm{HoH}$ can, for example, show the connection of a planned study to a body of theory, explicate and visualize the complexity of ideas implicitly included in a major hypothesis, or develop a research program around an overarching idea. 

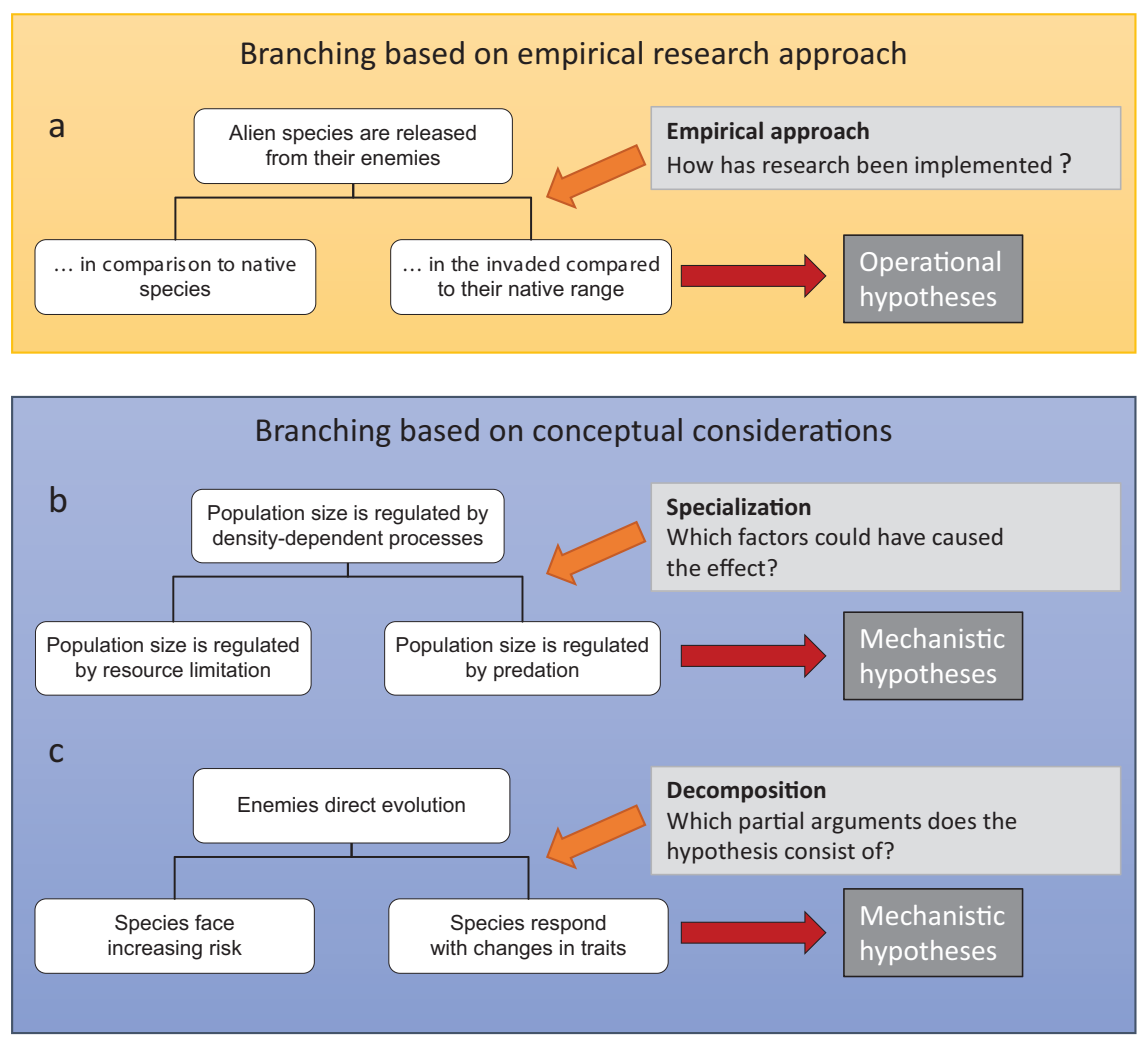

Figure 2. Three different types of branching in a hierarchy of hypotheses. The branching example shown in (a) is inspired by example 1 in the main text, (b) by example 2 (see also figure $3 b$ ), and (c) by example 3 (see also figure 4).

Step 3b. The hierarchical approach can additionally be used to connect the $\mathrm{HoH}$ developed in step 2 to a related body of theory. For example, Heger and colleagues (2013) suggested that the existing $\mathrm{HoH}$ on the enemy release hypothesis (see example 1 below) was conceptually connected to another well-known hypothesis-the novel weapons hypothesis. As a common overarching hypothesis addressing the question why species can successfully establish and spread outside of their native range, they suggested the "lack of eco-evolutionary experience hypothesis"; the enemy release and the novel weapons hypotheses are considered subhypotheses of this overarching hypothesis. This optional step can therefore help to create missing links within a discipline or even across disciplines.

Performing this step requires the study of the related body of theory, looking for conceptual overlaps and overarching topics. It may turn out that hypotheses, concepts, and ideas exist that are conceptually linked to the focal overarching hypothesis but that these links are nonhierarchical. In these cases, it can be useful to build hypothesis networks and apply clustering techniques to identify

Step 3a. If the aim is to identify research gaps, or to assess the generality or range of applicability of a major hypothesis, however, a further step must be taken (figure 1, step 3a): The $\mathrm{HoH}$ needs to be linked to empirical data. In previous studies (e.g., Jeschke and Heger 2018a), this step was done by assigning empirical studies to the subhypotheses they addressed and assessing the level of supporting evidence for the predictions derived from each hypothesis or subhypothesis. This assignment of studies to subhypotheses can be done either by using expert judgment or by applying machine learning algorithms (for further details, see Heger and Jeschke 2014, Jeschke and Heger 2018a, Ryo et al. 2019). Depending on the research question, the available resources and the structure of the data, the level of evidence can be assessed for each subhypothesis as well as for the higher-level hypotheses and can then be compared across subhypotheses. Such a comparison can provide information on the generality of an overarching hypothesis (i.e., its unifying power and breadth of applicability) or on the range of conditions under which a mechanism applies (see supplemental table S2 for examples). Before an $\mathrm{HoH}$ organizing theory is connected to empirical evidence, it will be necessary in most cases to include operational hypotheses at the lower levels, specifying, for example, different possible experimental approaches. underlying structures (see, e.g., Enders et al. 2020). This step can also be applied in cases in which the $\mathrm{HoH}$ has been built to organize evidence.

Step 4. Once the $\mathrm{HoH}$ is finalized, it can be published in order to enter the public domain and facilitate the advancement of the methodology and theory development. For the future, we envision a platform for the publication of $\mathrm{HoHs}$ to make the structured representations of research topics available not only via the common path of journal publications. The webpage www.hi-knowledge.org (Jeschke et al. 2018b) is a first step in this direction and is planned to allow for the upload of results in the future.

\section{Application of the HoH approach: Three examples}

We will now exemplify the process of creating an $\mathrm{HoH}$. The first example starts with a diverse set of empirical tests addressing one overarching hypothesis (i.e., with the aim to organize evidence), whereas the second and third examples start with conceptual considerations on how different aspects are linked to one overarching hypothesis (in the present article, the aim is to organize theory).

Example 1: The enemy release hypothesis as a hierarchy. The first published study showing a detailed version of an $\mathrm{HoH}$ focused on the enemy release hypothesis (Heger and 
Jeschke 2014). This is a prominent hypothesis in invasion biology (Enders et al. 2018). With respect to the research question of why certain species become invasive-that is, why they establish and spread in a new range-it posits, "The absence of enemies is a cause of invasion success" (e.g., Keane and Crawley 2002). With a systematic literature review, Heger and Jeschke (2014) identified studies addressing this hypothesis. This review revealed that the hypothesis has been tested in many different ways. After screening the empirical tests with a specific focus on which research approach had been used, the authors decided to use three branching criteria: the indicator for enemy release (actual damage, infestation with enemies or performance of the invader); the type of comparison (alien versus natives, aliens in native versus invaded range or invasive versus noninvasive aliens); and the type of enemies (specialists or generalists). On the basis of these criteria, Heger and Jeschke created a hierarchically organized representation of the hypothesis's multiple aspects. The order in which the three criteria were applied to create the hierarchy in this case was based on practical considerations. Empirical studies providing evidence were then assigned to the respective branch of the corresponding hierarchy to reveal specific subhypotheses that were more and others that were less supported (Heger and Jeschke 2014).

In later publications, Heger and Jeschke suggested some optional refinements of the original approach (Heger and Jeschke 2018b, 2018c). One of the suggestions was to distinguish between mechanistic hypotheses (originally termed working hypotheses) and operational hypotheses as different forms of subhypotheses when building the hierarchy. Mechanistic hypotheses serve the purpose of refining the broad, overarching idea in a conceptual sense (figure $2 \mathrm{~b}$ and 2c), whereas operational hypotheses refine the hypotheses by accounting for the diversity of study approaches (figure 2a).

The enemy release hypothesis example indicates that it can be useful to apply different types of branching criteria within one study. Heger and Jeschke (2014) looked for helpful ways of grouping diverse empirical tests. Some of the branches they decided to create were based on differences in the research methods, such as the distinction between comparisons of aliens versus natives, and comparisons of aliens in their native versus the invaded range (figure 2a). Other branches explicate complementary partial arguments contained in the major hypothesis: Studies in which the researchers asked whether aliens are confronted with fewer enemies were separated from those in which they asked whether aliens that are released show enhanced performance.

In this example, the $\mathrm{HoH}$ approach was used to organize evidence and therefore to expose the variety of manifestations of the enemy release hypothesis and to display the level of evidence for each branch of the $\mathrm{HoH}$ (see Heger and Jeschke 2018b and supplemental table S2 for an interpretation of the results).
Example 2: Illustrating the potential drivers of the snowshoe hareCanadian lynx population cycles. Understanding and predicting the spatiotemporal dynamics of populations is one of ecology's central goals (Sutherland et al. 2013), and population ecology has a long tradition of trying to understand causes for observed patterns in population dynamics. However, research efforts do not always produce clear conclusions, and often lead to competing explanatory hypotheses. A good example, which has been popularized through textbooks, is the 8-11-year synchronized population cycles of the snowshoe hare (Lepus americanus) and the Canadian lynx (Lynx canadensis; figure 3a). From eighteenth- to nineteenth-century fur trapping records across the North American boreal and northern temperate forests, it has been known that predator (lynx) and prey (hare) exhibit broadly synchronous population cycles. Research since the late 1930s (MacLulich 1937, Elton and Nicholson 1942) has tried to answer the question how these patterns are produced. A linear food chain of producer (vegetation) - primary consumer or prey (snowshoe hares) - secondary consumer or predator (Canadian lynx) proved too simplistic as an explanation (Stenseth et al. 1997). Instead, multiple drivers could have been responsible, resulting in the development of multiple competing explanations (Oli et al. 2020).

In the present article, we created an $\mathrm{HoH}$ to organize the current suggestions on what drives the snowshoe hare-lynx cycle (figure $3 \mathrm{~b}$ ). The aim of this exercise is to visualize conceptual connections rooted in current population ecological theory and, therefore, to enhance understanding of the complexity of involved processes.

A major hypothesis in population ecology is that populations are regulated by the interaction between biotic and abiotic factors. This regulation can either happen through processes coupled with the density of the focal organisms (density-dependent processes) or through density-independent processes, such as variability in environmental conditions or disturbances. This conceptual distinction can be used to branch out multiple mechanistic hypotheses that specify particular hypothetical mechanisms inducing the observed cycles. For example, potential drivers of the harelynx cycles include density-dependent mechanisms linked to bottom-up resource limitation and top-down predation, and density-independent mechanisms related to 10-year sun spot cycles. Figure $3 \mathrm{~b}$ also summarizes the kind of experiments that have been performed and how they relate to the corresponding mechanistic hypotheses. For example, food supplementation and fertilization experiments were used to test the resource limitation hypothesis and predator exclusion experiments to test the hypothesis that hare cycles are induced by predator abundance. Figure $3 b$ therefore highlights why it can be useful to apply very different types of experiments to test one broad overarching hypothesis.

The experiments that have been performed suggest that the predator-prey cycles result from an interaction between predation and food supplies combined with other modifying factors including social stress, disease and parasitism (Krebs 

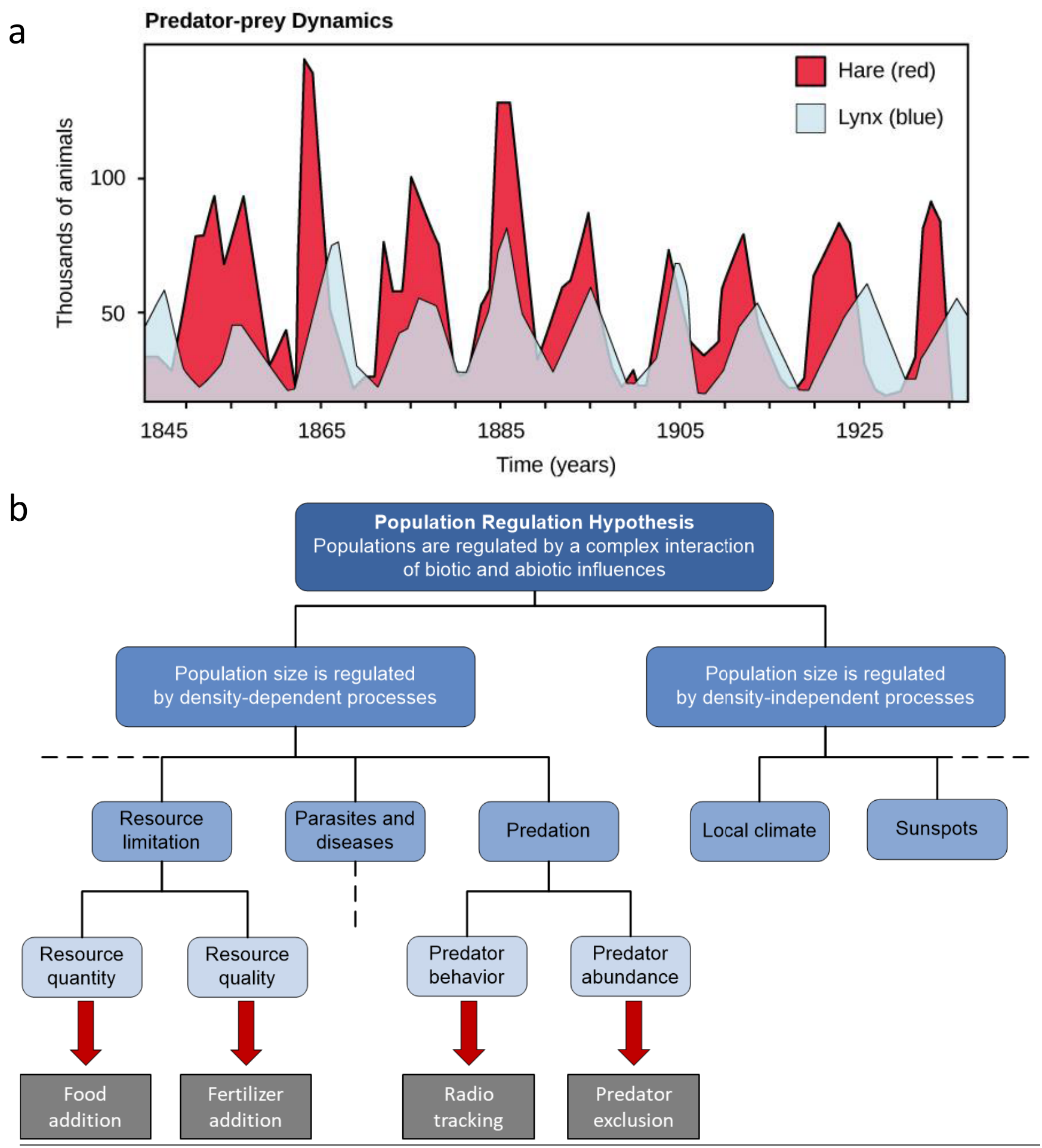

Figure 3. (a) The population cycle of snowshoe hare and Canadian lynx and (b) a hierarchy of hypotheses illustrating its potential drivers. The hypotheses (blue boxes) branch from the overarching hypothesis into more and more precise mechanistic hypotheses and are confronted with empirical tests (arrows leading to grey boxes) at lower levels of the hierarchy. The broken lines indicate where the hierarchy may be extended. Sources: The figure is based on the summary of snowshoe hare-Canadian lynx research (Krebs et al. 2001, Krebs et al. 2018 and references therein). Panel (a) is reprinted with permission from OpenStax Biology, Chapter 45.6 Community Ecology, Rice University Publishers, Creative Commons Attribution License (by 4.0). 
et al. 2001, 2018). Other experiments can be envisioned to test additional hypotheses, such as snow-removal experiments to test whether an increase in winter snow, induced by changed sun spot activity, causes food shortages and high hare mortality (Krebs et al. 2018).

In this example, alternate hypotheses are visually contrasted, and the different experiments that have been done are linked to the nested structure of possible drivers. This allows one to intuitively grasp the conceptual contribution of evidence stemming from each experiment to the overall explanation of the pattern. In a next step, quantitative results from these experiments could be summarized and displayed as well-for example, applying formal meta-analyses to summarize and display evidence stemming from each type of experiment. This example highlights how hierarchically structuring hypotheses can help to visually organize ideas about which drivers potentially cause a pattern in a complex system (for a comparison, see figure 11 in Krebs et al. 2018).

Example 3: The escalation hypothesis of evolution. The escalation hypothesis is a prominent hypothesis in evolutionary biology. In response to the question why species often seem to be well adapted to their biotic environment, it states that enemies are predominant agents of natural selection, and that enduring interactions with enemies brings about long-term evolutionary trends in the morphology, behavior, and distribution of organisms. Escalation, however, is an intrinsically costly process that can proceed only as long as resources are both available and accessible. Since the publication of Vermeij's book Evolution and Escalation in 1987, which is usually considered the start of the respective modern research program, escalation has represented anything but a fixed theory in its structure or content. The growth of escalation studies has led to the development of an increasing number of specific subhypotheses derived from Vermeij's original formulation and therefore to an expansion of the theoretical domain of the escalation hypothesis. Escalation has been supported by some tests but questioned by others.

Similar as in example 2, an $\mathrm{HoH}$ can contribute to conceptual clarity by structuring the diversity of escalation ideas that have been proposed (figure 4; Dietl 2015). To create the $\mathrm{HoH}$ for the escalation hypothesis, instead of assembling empirical studies that have tested it, Dietl (2015) went through the conceptual exercise of arranging existing escalation ideas on the basis of expert knowledge.

In its most generalized formulation-that is, "enemies direct evolution"-the escalation hypothesis can be situated at the top of a branch (figure 4) along with other hypotheses positing the importance of interaction-related adaptation, such as Van Valen's (1973) Red Queen hypothesis and hypotheses derived from Thompson's (2005) geographic mosaic theory of coevolution. Vermeij's original (1987) formulation of the hypothesis of escalation is actually composed of two separate testable propositions: "Biological hazards due to competitors and predators have become more severe over the course of time in physically comparable habitats" (p. 49 in Vermeij 1987) and "traits that enhance the competitive and antipredatory capacities of individual organisms have increased in incidence and in degree of expression over the course of time within physically similar habitats" (p. 49 in Vermeij 1987). As is the case with other composite hypotheses, these ideas must be singled out before the overarching idea can be unambiguously tested. This requirement creates a natural branching point in the escalation $\mathrm{HoH}$, the risk and response subhypotheses (figure 4).

Other lower-level hypotheses and aspects of the risk and response subhypotheses are possible. The risk side of the $\mathrm{HoH}$ can be further branched into subhypotheses suggesting either that the enemies evolved enhanced traits through time (e.g., allowing for greater effectiveness in prey capture) or that interaction intensity has increased through time (e.g., because of greater abundance or power of predators; figure 4). The response side of the $\mathrm{HoH}$ also can be further branched into several subhypotheses (all addressed by Vermeij 1987). In particular, species' responses could take the form of a trend toward more rapid exploitation of resources through time, an increased emphasis on traits that enable individuals to combat or interfere with competitors, a trend toward reduced detectability of prey through time, a trend of increased mobility (that is, active escape defense) through time, or an increase in the development of armor (or passive defense) through time. Arranging these different options of how escalation can manifest in boxes connected to a hierarchical structure helps to gain an overview. The depiction of subhypotheses in separate boxes does not indicate that the authors believe there is no interaction possible among these factors. For example, the evolution of enhanced traits may lead to an increase in interaction intensity. The presented $\mathrm{HoH}$ should be viewed only as one way to organize theory. It puts emphasis on the upward connections of subhypotheses to more general hypotheses. If the focus is more on interactions among different factors, other graphical and conceptual approaches may be more helpful (e.g., causal networks; for an example, see Gurevitch et al. 2011).

The HoH shown in figure 4 can be used as a conceptual backbone for further work in this field. Also, it can be related to existing evidence. This $\mathrm{HoH}$ will allow identification of data gaps and an understanding of which branches of the tree receive support by empirical work and therefore should be considered important components of escalation theory.

\section{Strengths and limits of the HoH approach}

The $\mathrm{HoH}$ approach can help to organize theory, to organize evidence, and to conceptualize and visualize connections of evidence to theory. Previously published examples of $\mathrm{HoHs}$ (e.g., Jeschke and Heger 2018a) and example 1 given above demonstrate its usefulness for organizing evidence, for pointing out important differences among subhypotheses and for conceptually and graphically connecting empirical results to a broader theoretical idea. Such an $\mathrm{HoH}$ can make the rationale underlying a specific study explicit and can 


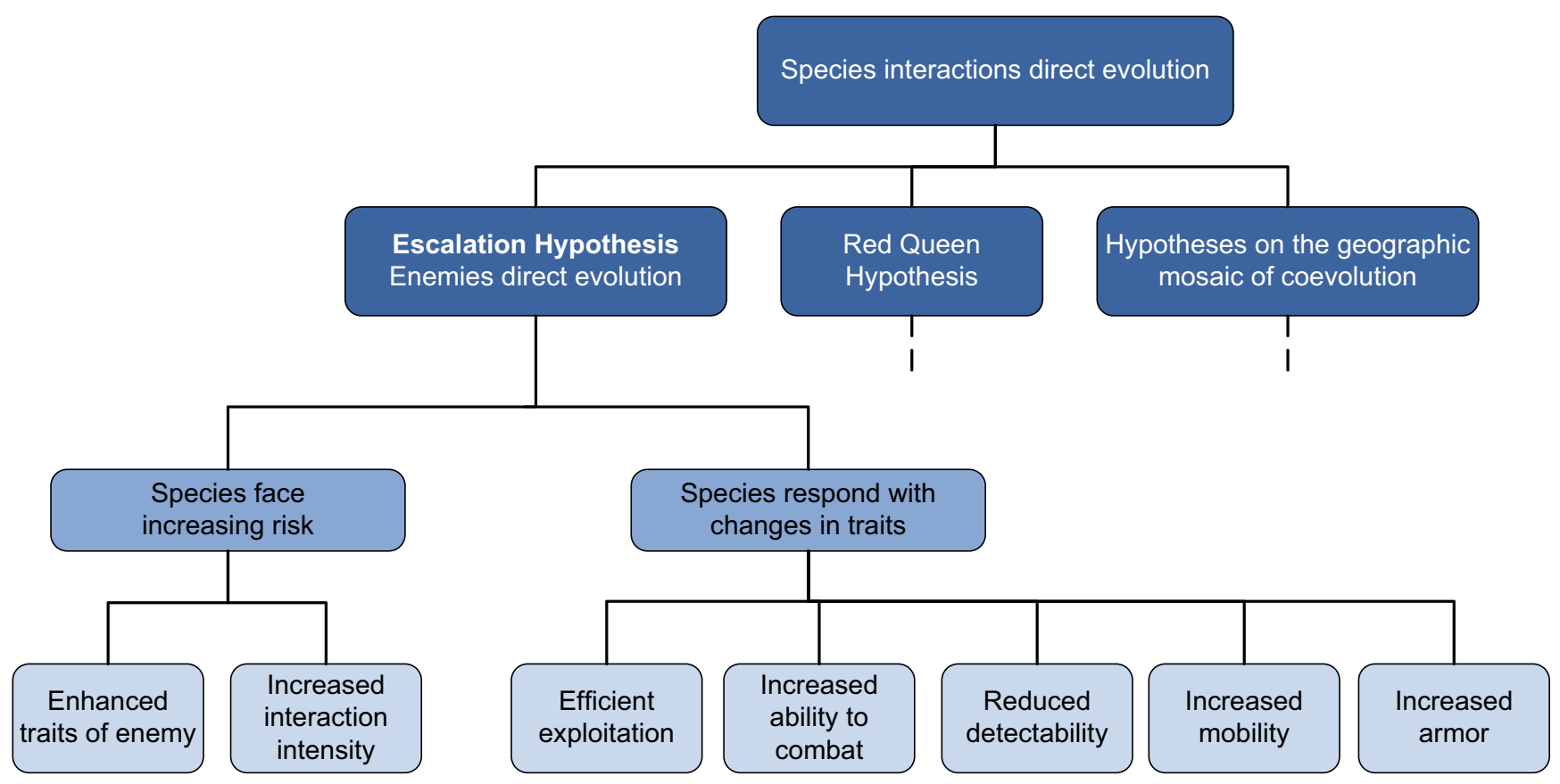

Figure 4. A hierarchy of hypotheses for the escalation hypothesis in evolutionary biology. The broken lines indicate where the hierarchy may be extended.

elucidate the conceptual connection of the study to a concrete theoretical background.

Applying the $\mathrm{HoH}$ approach can also help disclose knowledge gaps and biases (Braga et al. 2018) and can help reveal which research approaches have been used to assess an overarching idea (for examples, see Jeschke and Heger 2018a; other methods can be used to reach these aims tooe.g., systematic maps; Pullin et al. 2016, Collaboration for Environmental Evidence 2018). On the basis of such information, future research can be focused on especially promising areas or methods.

Besides such descriptive applications, the $\mathrm{HoH}$ approach can be combined with evidence assessment techniques (step 3a in figure 1). It can help to analyze the level of evidence for subhypotheses and therefore deliver the basis for discussing their usefulness and range of applicability (table S2; Jeschke and Heger 2018). Recent studies demonstrate that this kind of application can be useful for research outside of ecology as well-for example, in biomedical research (Bartram and Jeschke 2019) or even in a distant field like company management research (Wu et al. 2019).

We did not detail in the present article how the confrontation of hypotheses with evidence in an $\mathrm{HoH}$ can be done, but in previous work it was shown that this step can deliver the basis for enhancing theory. For example, the $\mathrm{HoH}$-based literature analyses presented in Jeschke and Heger (2018a) showed that several major hypotheses in invasion biology are only weakly supported by evidence. The authors consequently suggested to reformulate them (Jeschke and Heger 2018b) and to explicitly assess their range of applicability (Heger and Jeschke 2018a). Because an $\mathrm{HoH}$ visually connects data and theory, the approach motivates one to feed empirical results back into theory and, therefore, use them for improving theory. It is our vision that in the future, theory development in ecology and evolution could largely profit from a regular application of the $\mathrm{HoH}$ approach. Steps to improve theory can include highlighting strongly supported subhypotheses, pointing out hypotheses with low unification power and breadth of applicability, shedding light on previously unnoticed connections, and revealing gaps in research.

The examples on the hare-lynx cycles and the escalation hypothesis showed that the $\mathrm{HoH}$ approach can also guide theory-driven reasoning in both the ecological and evolutionary domains, respectively. That is, the $\mathrm{HoH}$ approach can allow the reconsideration and reorganization of conceptual ideas without directly referring to data. Major hypotheses or research questions are usually composed of several elements, and above, we suggest how these elements can be exposed and visualized (figure $2 \mathrm{~b}$ and $2 \mathrm{c}$ ). In this way, applying the $\mathrm{HoH}$ approach can help to enhance conceptual clarity by displaying different meanings and components of broad concepts. Conceptual clarity is not only useful to avoid miscommunication or misinterpretation of empirical results, but we expect that it will also facilitate theory development by enhancing accurate thinking and argumentation.

In addition, the nested, hierarchical structure invites looking for connections upward: Figure 4 shows the escalation hypothesis as one variant of an even broader hypothesis, positing that "Species interactions direct evolution." This in turn can enhance the future search for patterns and mechanisms across unconnected study fields. A respective 
example can be found in Schulz and colleagues (2019). In that article, the authors used the $\mathrm{HoH}$ approach to organize twelve hypotheses each addressing the roles that antagonists play during species invasions. By grouping the hypotheses in a hierarchically nested way, Schulz and colleagues showed their conceptual relatedness, which had not been demonstrated before.

In the future, the $\mathrm{HoH}$ approach could also be used for creating interdisciplinary links. There are many research questions that are being addressed in several research areas in parallel, using different approaches and addressing different aspects of the overall question. In an $\mathrm{HoH}$, such connections could be revealed. Heger and colleagues (2019) suggested a future application of the $\mathrm{HoH}$ approach for organizing and structuring research on effects of global change on organisms, communities, and ecosystems. Under the broad header of "ecological novelty," more specific research questions addressed in various disciplines (e.g., climate change research, biodiversity research, urban ecology, restoration ecology, evolutionary ecology, microbial ecology) could be organized and therefore conceptually connected.

Importantly, the $\mathrm{HoH}$ approach can be easily combined with existing synthesis tools. For example, as was outlined above and in figure 1, a systematic literature review can be used to identify and structure primary studies to be used for building an $\mathrm{HoH}$. Statistical approaches, such as machine learning, can be used to optimize branching with respect to levels of evidence (Ryo et al. 2019), and empirical data structured in an $\mathrm{HoH}$ can be analyzed with formal meta-analysis-for example, separately for each subhypothesis (Jeschke and Pyšek 2018). In future applications, an $\mathrm{HoH}$ could also be used to visualize the results of a research-weaving process, in which systematic mapping is combined with bibliometric approaches (Nakagawa et al. 2019). Furthermore, HoHs can be linked to a larger network. An example is the website https://hi-knowledge.org/invasion-biology/ (Jeschke et al. 2018b) where the conceptual connections of 12 major hypotheses of invasion ecology are displayed as a hierarchical network. We believe that the combination of $\mathrm{HoH}$ with other knowledge synthesis tools, such as Venn diagrams, ontologies, controlled vocabularies, and systematic maps, can be useful as well and should be explored in the future.

We emphasize, however, that the $\mathrm{HoH}$ method is by far no panacea for managing complexity. Not all topics interesting for scientific inquiry can be organized hierarchically, and imposing a hierarchy may even lead to wrong conclusions, thus actually hindering theory development. For example, to focus a conceptual synthesis on one major overarching hypothesis may conceal that other factors not addressed by this single hypothesis have a major effect on underlying processes as well. Evidence assessed with respect to this one hypothesis can in such cases only be used to derive partial explanations, whereas for a more complete understanding of the underlying processes, interactions with other factors need to be considered. Furthermore, displaying interacting aspects of a system as discrete entities within a hierarchy can obfuscate the true dynamics of a system.

In our three examples-the enemy release hypothesis, the hare-lynx cycles, and the escalation hypothesis (figures 3 and 4)-connections between the different levels of the hierarchies do not necessarily depict causal relationships. Also, the fact that multicausality is ubiquitous in ecological systems is not covered. It has been argued that approaches directly focusing on explicating causal relationships and multicausality could be more helpful for advancing theory (Scheiner and Fox 2018). The HoH approach is currently primarily a tool to provide conceptual structure. We suggest that revealing causal networks and multicausalities represent additional objectives and regard them as important aims also for further developing the $\mathrm{HoH}$ approach. Combining existing approaches for revealing causal relationships (e.g., Eco Evidence, Norris et al. 2012, or CADDIS, www.epa.gov/caddis) with the $\mathrm{HoH}$ approach seems to be a promising path forward. Also, a future aim could be to develop a version of the $\mathrm{HoH}$ approach with enhanced formalization, allowing different kinds of relationships among subhypotheses to be disclosed (e.g., applying semantic web methods. Such a formalized version of the $\mathrm{HoH}$ approach could be used for scrutinizing the logical structure of hypotheses (e.g., compatibility and incompatibility of subhypotheses) and identifying inevitable interdependencies (e.g., likelihood of cooccurrence of evidence along two branches).

The guidelines on how to build an $\mathrm{HoH}$ presented above and in figures 1 and 2 will help to increase the reproducibility of the process. Full reproducibility is unlikely to be reached for most applications because researchers need to make individual choices. For example, step 1 involves creative reasoning and may therefore potentially lead to differing results if repeated by different researchers. The process of creating an $\mathrm{HoH}$ can therefore lead to a whole set of outcomes. Usually, there will be not one single $\mathrm{HoH}$ that is the one "correct" answer to the research questions. Certain steps of the process can be automated using artificial intelligence, such as with the use of decision-tree algorithms to enhance reproducibility (Ryo et al. 2019). But even if such techniques are applied, the choice of which information is fed into the algorithms is made by a researcher. We suggest that this ambiguity should not be considered a flaw of the method, but instead an important and necessary concession to creativity, offering the chance to closely match the outcome of the process to the concrete requirements of the research project. Also, it should be noted that other approaches for knowledge synthesis do not necessarily yield reproducible results either, not even formal meta-analysis (de Vrieze 2018).

\section{Conclusions}

The current emphasis on statistical approaches for synthesizing evidence with the purpose of facilitating decision making in environmental management and nature conservation is undoubtedly important and necessary. However, 
knowledge and understanding of ecological systems would profit largely if results from empirical studies would in addition, and on a regular basis, be used to improve theory. With this contribution, we present one possibility for creating close links between evidence and theory, and we hope to stimulate future studies that feed results from case studies back into theory. Our goal is to motivate more conceptual work aimed at refining major hypotheses on how complex systems work. Above, we provided examples for how to develop a nuanced representation of major hypotheses, focusing on their mechanistic components.

Ecological systems are highly complex, and therefore, the theories describing them typically need to incorporate complexity. Nested, hierarchical structures in our view represent one possible path forward, because they allow zooming in and out and, therefore, moving between different levels of complexity. We propose that alternative tools such as causal networks should be further developed for application in ecology and evolution as well. Combining complementary conceptual tools would in our view be most promising for an efficient enhancement of knowledge and understanding in ecology.

\section{Acknowledgments}

The ideas presented in this article were developed during the workshop "The hierarchy-of-hypotheses approach: Exploring its potential for structuring and analyzing theory, research, and evidence across disciplines," 19-21 July 2017, and refined during the workshop "Research synthesis based on the hierarchy-of-hypotheses approach," 10-12 October 2018, both in Hanover, Germany. We thank William Bausman, Adam Clark, Francesco DePrentis, Carsten Dormann, Alexandra Erfmeier, Gordon Fox, Jeremy Fox, James Griesemer, Volker Grimm, Thierry Hanser, Frank Havemann, Yuval Itescu, Marie Kaiser, Julia Koricheva, Peter Kraker, Ingolf Kühn, Andrew Latimer, Chunlong Liu, Bertram Ludäscher, Klaus Mainzer, Elijah Millgram, Bob O’Hara, Masahiro Ryo, Raphael Scholl, Gerhard Schurz, Philip Stephens, Koen van Benthem and Meike Wittman for participating in our lively discussions and Alkistis Elliot-Graves and Birgitta König-Ries for help with refining terminology. Furthermore, we thank Sam Scheiner and five anonymous reviewers for comments that helped to improve the manuscript. The workshops were funded by Volkswagen Foundation (Az 92,807 and 94,246). TH, CAA, ME, PG, ADS, and JMJ received funding from German Federal Ministry of Education and Research within the Collaborative Project "Bridging in Biodiversity Science" (grant no. 01LC1501A). ME additionally received funding from the Foundation of German Business, JMJ from the Deutsche Forschungsgemeinschaft (grants no. JE 288/9-1 and JE 288/9-2), and IB from German Federal Ministry of Education and Research (grant no. FKZ 01GP1710). CJL was supported by a grant from The Natural Sciences and Engineering Research Council of Canada and in-kind synthesis support from the US National Center for Ecological Analysis and Synthesis. LGA was supported by the Spanish
Ministry of Science, Innovation, and Universities through project no. CGL2014-56,739-R, and RRB received funding from the Brazilian National Council for Scientific and Technological Development (process no. 152,289/2018-6).

\section{Supplemental material}

Supplemental material is available at BIOSCI online.

\section{References cited}

Bartram I, Jeschke JM. 2019. Do cancer stem cells exist? A pilot study combining a systematic review with the hierarchy-of-hypotheses approach. PLOS ONE 14: e0225898.

Braga RR, Gómez-Aparicio L, Heger T, Vitule JRS, Jeschke JM. 2018. Structuring evidence for invasional meltdown: Broad support but with biases and gaps. Biological Invasions 20: 923-936.

Collaboration for Environmental Evidence. 2018. Guidelines and Standards for Evidence Synthesis in Environmental Management, version 5.0. Collaboration for Environmental Evidence. www.environmentalevidence.org/information-for-authors.

Cook CN, Nichols SJ, Webb JA, Fuller RA, Richards RM. 2017. Simplifying the selection of evidence synthesis methods to inform environmental decisions: A guide for decision makers and scientists. Biological Conservation 213: 135-145.

de Vrieze J. 2018. The metawars. Science 361: 1184-1188.

Dicks LV, et al. 2017. Knowledge Synthesis for Environmental Decisions: An Evaluation of Existing Methods, and Guidance for Their Selection, Use, and Development. EKLIPSE Project.

Diefenderfer HL, Johnson GE, Thom RM, Bunenau KE, Weitkamp LA, Woodley CM, Borde AB, Kropp RK. 2016. Evidence-based evaluation of the cumulative effects of ecosystem restoration. Ecosphere 7: e01242.

Dietl GP. 2015. Evaluating the strength of escalation as a research program. Geological Society of America Abstracts with Programs 47: 427

Elton C, Nicholson M. 1942. The ten-year cycle in numbers of lynx in Canada. Journal of Animal Ecology 11: 215-244.

Enders M, Hütt M-T, Jeschke JM. 2018. Drawing a map of invasion biology based on a network of hypotheses. Ecosphere 9: e02146.

Enders M, et al. 2020. A conceptual map of invasion biology: Integrating hypotheses into a consensus network. Global Ecology and Biogeography 29: 978-999.

Farji-Brener AG, Amador-Vargas S. 2018. Hierarchy of hypotheses or hierarchy of predictions? Clarifying key concepts in ecological research. Pages 19-22 in Jeschke JM, Heger T, eds. Invasion Biology: Hypotheses and Evidence. CAB International.

Giere RN, Bickle J, Mauldin R. 2005. Understanding Scientific Reasoning, 5 th ed. Wadsworth Cengage Learning.

Grace J, Anderson T, Olff H, Scheiner S. 2010. On the specification of structural equation models for ecological systems. Ecological Monographs 80: 67-87.

Griesemer JR. 2013. Formalization and the meaning of "theory" in the inexact biological sciences. Biological Theory 7: 298-310.

Griesemer J. 2018. Mapping theoretical and evidential landscapes in ecological science: Levin's virtue trade-off and the hierarchy-of-hypotheses approach. Pages 23-29 in Jeschke JM, Heger T, eds. Invasion Biology: Hypotheses and Evidence. CAB International.

Gurevitch J, Fox GA, Wardle GM, Inderjit, Taub D. 2011. Emergent insights from the synthesis of conceptual frameworks for biological invasions. Ecology Letters 14: 407-418.

Haddaway NR, Macura B, Whaley P, Pullin AS. 2018. ROSES: Reporting standards for systematic evidence syntheses: Pro forma, flow-diagram and descriptive summary of the plan and conduct of environmental systematic reviews and systematic maps. Environmental Evidence 7: 7.

Heger T, Jeschke JM. 2014. The enemy release hypothesis as a hierarchy of hypotheses. Oikos 123: 741-750. 
Heger T, Jeschke JM. 2018a. Conclusions and outlook. Pages 167-172 in Jeschke JM, Heger T, eds. Invasion Biology: Hypotheses and Evidence. CAB International.

Heger T, Jeschke JM. 2018b. Enemy release hypothesis. Pages 92-102 in Jeschke JM, Heger T, eds. Invasion Biology: Hypotheses and Evidence. CAB International.

Heger T, Jeschke JM. 2018c. The hierarchy-of-hypotheses approach updated: A toolbox for structuring and analysing theory, research, and evidence. Pages 38-48 in Jeschke JM, Heger T, eds. Invasion Biology: Hypotheses and Evidence. CAB International.

Heger T, et al. 2013. Conceptual frameworks and methods for advancing invasion ecology. Ambio 42: 527-540.

Heger T, et al. 2019. Towards an integrative, eco-evolutionary understanding of ecological novelty: Studying and communicating interlinked effects of global change. BioScience 69: 888-899.

Howick J. 2011. The Philosophy of Evidence-based Medicine. Wiley-Blackwell.

Jeltsch F, et al. 2013. How can we bring together empiricists and modelers in functional biodiversity research? Basic and Applied Ecology 14: 93-101.

Jeschke JM, Heger T, eds. 2018a. Invasion Biology: Hypotheses and Evidence. CAB International.

Jeschke JM, Heger T, eds. 2018b. Synthesis. Pages 157-166 in Jeschke JM, Heger T, eds. Invasion Biology. Hypotheses and Evidence. CAB International.

Jeschke JM, Pyšek P. 2018. Tens rule. Pages 124-132 in Jeschke JM, Heger T, eds. Invasion Biology: Hypotheses and Evidence. CAB International.

Jeschke JM, Gómez Aparicio L, Haider S, Heger T, Lortie CJ, Pyšek P, Strayer DL. 2012. Support for major hypotheses in invasion biology is uneven and declining. NeoBiota 14: 1-20.

Jeschke JM, Debille S, Lortie CJ. 2018a. Biotic resistance and island susceptibility hypotheses. Pages 60-70 in Jeschke JM, Heger T, eds. Invasion Biology: Hypotheses and Evidence. CAB International.

Jeschke JM, Enders M, Bagni M, Jeschke P, Zimmermann M, Heger T. 2018b. Hi Knowledge. Hi-Knowledge.org. www.hi-knowledge.org/ invasion-biology

Jeschke JM, Lokatis S, Bartram I, Tockner K. 2019. Knowledge in the dark: Scientific challenges and ways forward. FACETS 4: 1-19.

Keane RM, Crawley MJ. 2002. Exotic plant invasions and the enemy release hypothesis. Trends in Ecology and Evolution 17: 164-170.

Koricheva J, Gurevitch J, Mengersen K, eds. 2013. Handbook of Metaanalysis in Ecology and Evolution. Princeton University Press.

Krebs CJ, Boonstra R, Boutin S. 2018. Using experimentation to understand the 10-year snowshoe hare cycle in the boreal forest of North America. Journal of Animal Ecology 87: 87-100.

Krebs CJ, Boonstra R, Boutin S, Sinclair ARE. 2001. What drives the 10-year cycle of snowshow hares? BioScience 51: 25-35.

Lortie CJ. 2014. Formalized synthesis opportunities for ecology: Systematic reviews and meta-analyses. Oikos 123: 897-902.

MacLulich DA. 1937. Fluctuation in numbers of the varying hare (Lepus americanus). Univ Toronto Studies Biol Series 43: 1-136.

Moher D, Shamseer L, Clarke M, Ghersi D, Liberati A, Petticrew M, Shekelle P, Stewart LA, PRISMA-P Group. 2015. Preferred reporting items for systematic review and meta-analysis protocols (PRISMA-P) 2015 statement. Systematic Reviews 4: 1.

Mupepele A-C, Walsh JC, Sutherland WJ, Dormann CF. 2016. An evidence assessment tool for ecosystem services and conservation studies. Ecological Applications 26: 1295-1301.

Nakagawa S, Samarasinghe G, Haddaway NR, Westgate MJ, O’Dea RE, Noble DWA, Lagisz M. 2019. Research weaving: Visualizing the future of research synthesis. Trends in Ecology and Evolution 34: 224-238.

Nesshöver C, et al. 2016. The Network of Knowledge approach: Improving the science and society dialogue on biodiversity and ecosystem services in Europe. Biodiversity and Conservation 25: 1215-1233.

Norris RH, Webb JA, Nichols SJ, Stewardson MJ, Harrison ET. 2012. Analyzing cause and effect in environmental assessments: Using weighted evidence from the literature. Freshwater Science 31: 5-21.
Oli MK, Krebs CJ, Kenney AJ, Boonstra R, Boutin S, Hines JE. 2020 Demography of snowshoe hare population cycles. Ecology 101: e02969. doi:10.1002/ecy.2969

Pickett STA, Kolasa J, Jones CG. 2007. Ecological Understanding: The Nature of Theory and the Theory of Nature, 2nd ed. Academic Press.

Platt JR. 1964. Strong inference. Science 146: 347-353.

Pullin A, et al. 2016. Selecting appropriate methods of knowledge synthesis to inform biodiversity policy. Biodiversity and Conservation 25 $1285-1300$.

Ryo M, Jeschke JM, Rillig MC, Heger T. 2019. Machine learning with the hierarchy-of-hypotheses $(\mathrm{HoH})$ approach discovers novel pattern in studies on biological invasions. Research Synthesis Methods 11: 66-73 doi: $10.1002 /$ jrsm. 1363

Scheiner SM. 2013. The ecological literature, an idea-free distribution. Ecology Letters 16: 1421-1423.

Scheiner SM, Fox GA. 2018. A hierarchy of hypotheses or a network of models. Pages 30-37 in Jeschke JM, Heger T, eds. Invasion Biology: Hypotheses and Evidence. CAB International.

Schulz AN, Lucardi RD, Marsico TD. 2019. Successful invasions and failed biocontrol: The role of antagonistic species interactions. BioScience 69: 711-724.

Silvertown JW, Charlesworth D. 2001. Introduction to Plant Population Biology. Blackwell Scientific.

Stenseth NC, Falck W, Bjørnstad ON, Krebs CJ. 1997. Population regulation in snowshoe hare and Canadian lynx: Asymmetric food web configurations between hare and lynx. Proceedings of the National Academy of Sciences of the United States of America 94: 5147-5152.

Sutherland WJ. 2006. Predicting the ecological consequences of environmental change: A review of the methods. Journal of Applied Ecology 43: 599-616.

Sutherland WJ, et al. 2013. Identification of 100 fundamental ecological questions. Journal of Ecology 101: 58-67.

Thompson JN. 2005. The Geographic Mosaic of Coevolution. University of Chicago Press.

Van Valen L. 1973. A new evolutionary law. Evolutionary Theory 1: 1-30.

Vermeij GJ. 1987. Evolution and Escalation: An Ecological History of Life. Princeton University Press.

Wu L, Huang I-C, Huang W-C, Du P-L. 2019. Aligning organizational culture and operations strategy to improve innovation outcomes: An integrated perspective in organizational management. Journal of Organizational Change Management 32: 224-250. doi:10.1108/ JOCM-03-2018-0073

Tina Heger (t.heger@wzw.tum.de) is affiliated with the Department of Biodiversity Research and Systematic Botany and Alexis D. Synodinos is affiliated with the Department of Plant Ecology and Nature Conservation at the University of Potsdam, in Potsdam, Germany. Tina Heger and Kurt Jax are affiliated with the Department of Restoration Ecology at the Technical University of Munich, in Freising, Germany. Tina Heger, Carlos A. Aguilar-Trigueros, Martin Enders, Pierre Gras, Jonathan M. Jeschke, Sophie Lokatis, and Alexis Synodinos are affiliated with the Berlin-Brandenburg Institute of Advanced Biodiversity Research (BBIB), in Berlin, Germany. Carlos Aguilar, Isabelle Bartram, Martin Enders, Jonathan M. Jeschke, and Sophie Lokatis are affiliated with the Institute of Biology at Freie Universität Berlin, in Berlin, Germany. Martin Enders, Jonathan M. Jeschke, and Sophie Lokatis are also affiliated with the Leibniz Institute of Freshwater Ecology and Inland Fisheries (IGB), in Berlin, Germany. Pierre Gras is also affiliated with the Department of Ecological Dynamics at the Leibniz Institute for Zoo and Wildife Research (IZW), also in Berlin, Germany. Isabelle Bartram is affiliated with the Institute of Sociology, at the University of Freiburg, in Freiburg. Kurt Jax is also affiliated with the Department of Conservation Biology at the Helmholtz Centre for Environmental Research-UFZ, in Leipzig, Germany. Raul R. Braga is located at the Universidade Federal do Paraná, Laboratório de Ecologia e Conservação, in Curitiba, Brazil. Gregory P. Dietl has two affiliations: the Paleontological Research Institution and the Department of Earth and Atmospheric Sciences at Cornell University, in Ithaca, New York. David 
J. Gibson is affiliated with the School of Biological Sciences at Southern Illinois University Carbondale, in Carbondale, Illinois. Lorena GómezAparicio's affiliation is the Instituto de Recursos Naturales y Agrobiología de Sevilla, CSIC, LINCGlobal, in Sevilla, Spain. Christopher J. Lortie is affiliated with the Department of Biology at York University, in York, Canada, as well as with the National Center for Ecological Analysi and Synthesis, at the University of California Santa Barbara, in Santa Barbara, California. Anne-Christine Mupepele has two affiliations as well: the Chair of Nature Conservation and Landscape Ecology at the University of Freiburg, in Freiburg, and the Senckenberg Biodiversity and Climate Research Centre, in Frankfurt am Main, both in Germany.
Stefan Schindler is working at the Environment Agency Austria and the University of Vienna's Division of Conservation Biology, Vegetation, and Landscape Ecology, in Vienna, Austria, and his third affiliation is with Community Ecology and Conservation, at the Czech University of Life Sciences Prague, in Prague, Czech Republic. Finally, Jostein Starrfelt is affiliated with the University of Oslo's Centre for Ecological and Evolutionary Synthesis and with the Norwegian Scientific Committee for Food and Environment, Norwegian Institute of Public Health, both in Oslo, Norway. Alexis D. Synodinos is affiliated with the Centre for Biodiversity Theory and Modelling, Theoretical, and Experimental Ecology Station, CNRS, in Moulis, France. 